\title{
The Successful Treatment of Herniated Lumbar Discs That are Refractory to Repeated Epidural Steroid Injection by Using a Navigable Percutaneous Disc Decompression Device: A Case Series
}

This article was published in the following Dove Press journal:

Journal of Pain Research

\author{
Min Young Lee' \\ Mathieu \\ Boudier-Revéret (D) $^{2}$ \\ Hee Kyung $\mathrm{Cho}^{3}$ \\ Min Cheol Chang' \\ 'Department of Physical Medicine and \\ Rehabilitation, College of Medicine, \\ Yeungnam University, Namku, Taegu, \\ Republic of Korea; ${ }^{2}$ Department of \\ Physical Medicine and Rehabilitation, \\ Centre Hospitalier de l'Université de \\ Montréal, Montreal, QC, Canada; \\ ${ }^{3}$ Department of Rehabilitation Medicine, \\ Catholic University of Daegu School of \\ Medicine, Daegu, Republic of Korea
}

Correspondence: Min Cheol Chang Email wheel633@gmail.com

\begin{abstract}
Purpose: In most cases, lumbosacral radicular pain caused by herniated lumbar discs (HLDs) can be controlled with epidural steroid injections (ESIs). However, when the HLDs are large, the pain may not respond to ESIs. A navigable, percutaneous, disc decompression device has recently been developed to manage radicular pain that is secondary to HLD, which allows the wand tip to approach the herniated disc by rotating a control wheel. We performed a percutaneous disc decompression using the navigable percutaneous disc decompression device in two patients with a large HLD that did not respond to repeated ESIs.
\end{abstract}

Patients and Methods: Patients A and B are presented with scores of 7 and 8 on the numeric rating scale (NRS), respectively. Both had lumbosacral radicular pain due to right central HLDs at L4-5 and L5-S1, despite repeated ESIs. Percutaneous disc decompression was performed under $\mathrm{C}$-arm fluoroscopy. The wand was inserted through the introducer needle. Using the control wheel, we placed the needle tip on the posterolateral portion of the herniated disc. The radiofrequency current was applied to the herniated portion of the disc. The procedural time was 20-30 minutes.

Results: Neither of the patients reported adverse post-procedural effects. At their 1-week follow-up, patient A and B's NRS pain scores had reduced to 2 and 1, respectively. At their 2-year follow-up, patient A had mild pain (NRS 1), and patient B reported no pain.

Conclusion: The navigable percutaneous disc decompression device may be effective for pain alleviation in patients with lumbosacral radicular pain that is refractory to repeated ESIs.

Keywords: lumbosacral radicular pain, disc decompression, herniated lumbar disc

\section{Introduction}

Herniated lumbar discs (HLDs) are among the most commonly occurring musculoskeletal diseases that cause radiating pain in the lower extremities. ${ }^{1,2}$ The mechanisms underlying pain following HLD are chemical inflammation around the nerve roots and their mechanical compression. ${ }^{3}$ Chemical inflammation is the main mechanism that underlies radicular pain in most cases of HLD, and it can be successfully managed nonsurgically with interventions such as epidural steroid injections (ESIs) and oral medications. ${ }^{4,5}$ However, when the size of the herniated disc is large, the pain may not respond to these interventions, which makes decompression with surgery the most viable option. 
Minimally invasive disc decompression (MIDD) has compelling advantages over open surgery. Only one or two small-diameter (a few millimeters) skin punctures are required for MIDD; it minimally invades the skin, fascia, and muscles compared to open surgery. A smaller incision reduces the risk of adverse effects, such as blood loss and infections at the incision site, and promotes faster recovery after treatment. ${ }^{6}$ Additionally, local skin anesthesia is sufficient for MIDD, whereas general anesthesia should be performed for open surgery. However, previous studies have reported lower successful treatment rates with MIDD than that with open surgery, although MIDD is safer and less invasive. ${ }^{7-9}$

Various devices have been used for MIDD. During these procedures, the wand is usually inserted into the lumbar disc through the introducer needle and it vaporizes nuclear tissue using bipolar radiofrequency applied to a saline-conducting medium. However, this technique is limited because the wand tip cannot easily reach the herniated nucleus. ${ }^{6}$ This limitation may account for the reduced success rates of MIDD in controlling radicular pain due to HLD.

Compared with previous devices, the recently developed, navigable percutaneous disc decompression device has a wand tip that can be curved by rotating a control wheel; this allows the tip of the wand better access to the herniated disc, where it can apply the bipolar radiofrequency current (Figure 1). ${ }^{6}$ Its effectiveness in managing radicular pain following HLD has been previously reported. ${ }^{6,10}$ However, its effectiveness for HLD pain that is refractory to repeated ESIs and that requires open surgery has not been reported.

We report two cases of lumbosacral radicular pain following HLD that did not respond to repeated ESIs but were successfully managed using a navigable percutaneous disc decompression device.

\section{Case Presentations}

Written informed consent was obtained from the patients for publication of this case report as well as the accompanying images.

Two patients with lumbosacral radicular pain that was refractory to repeated ESIs were recruited for this study. Both patients provided informed content for participation in this research. The study was approved by the Institutional Review Board at Yeungnam University Hospital.
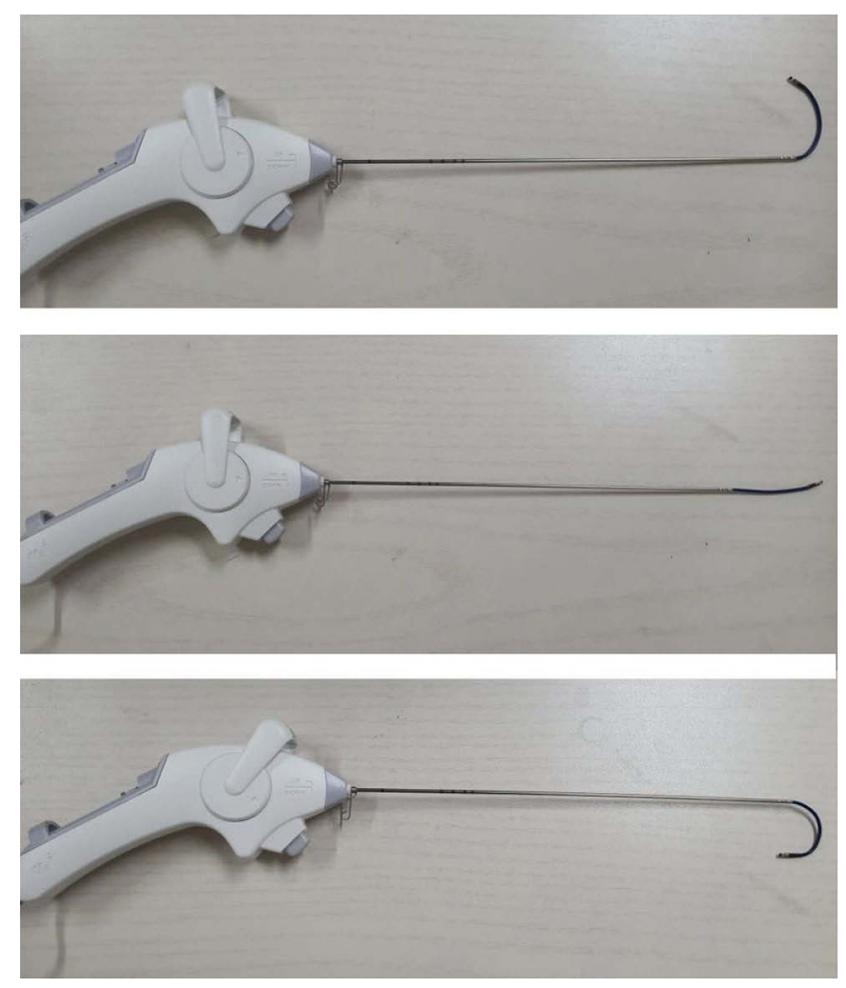

Figure I L'DISQ ${ }^{\circledR}$ 's wand, with its navigable tip. The tip of the wand can be curved to the desired angle by rotating the control wheel.

Patient A, a 48-year-old man, visited the spine center at the University Hospital with pain in the right buttock, lateral thigh, and calf, which had lasted for two weeks. The pain was piercing and tingling. The patient had a score of 7 out of 10 on the numeric rating scale (NRS). The pain was aggravated when he flexed his lower back. Upon physical examination, he had a positive straight legraising test (SLRT) result at 40 degrees on the right side. His right extensor hallucis longus had a Medical Research Council (MRC) score of 3, but no sensory deficits were observed. Lumbar magnetic resonance imaging (MRI) revealed a massive right central extrusion of the L4-5 intervertebral disc (Figure 2A). Before he visited our hospital, he had received three repeated transforaminal ESIs at the right L5 nerve root, as well as two repeated interlaminar ESIs at L4-5. However, no pain reduction was observed following each procedure. At his next visit to our hospital, we conducted a percutaneous disc decompression with radiofrequency energy using the navigable percutaneous disc decompression device (L'DISQ ${ }^{\circledR}, \mathrm{U}$

\& I Co., Uijeongbu, South Korea) (Figure 2A). The procedure was conducted under the guidance of fluoroscopy. The patient was in the prone position, and we marked the patient's skin approximately $13 \mathrm{~cm}$ from the 


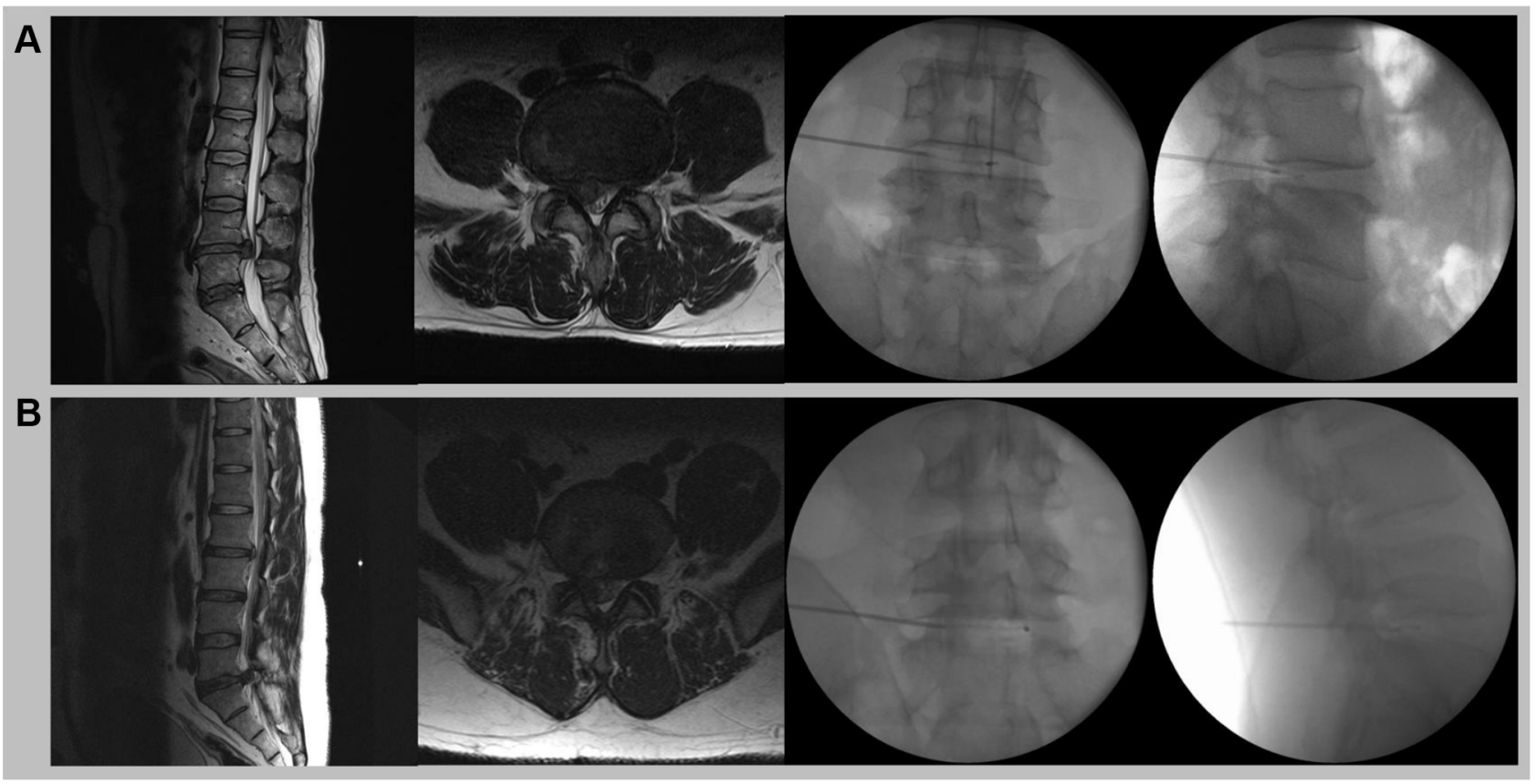

Figure 2 (A) Patient A; (Left) the sagittal and axial T2-weighted lumbar spine MRI shows a massive right central herniated lumbar disc at L4-5. (Right) The wand with a curved tip is inserted into the L4-5 intervertebral disc to perform the percutaneous disc decompression, and the tip is placed near the herniated disc. (B) Patient B; (Left) the sagittal and axial T2-weighted lumbar spine MRI reveals a large right central herniated lumbar disc at L5-SI. (Right) The wand is inserted into the L5-SI intervertebral disc with the tip placed near the herniated disc.

midline in order to guide the needle entry. The endplates of the target disc (L4-5) space were aligned. The C-arm was rotated ipsilaterally in order to position the lateral margin of the ipsilateral superior articular process. This was done at approximately $3 / 5$ of the distance across the vertebral body, as visualized in the oblique position (typically rotated 15 degrees from a zero-degree lateral projection). The C-arm was rotated into the contralateral side (left) of the disc herniation. After skin sterilization and anesthesia, we inserted a 16-gauge introducer needle, directing it gently toward the annular surface of the contralateral foramen (slightly lateral portion of the lateral edge of the superior articular process) using fluoroscopy. Once the disc surface was touched, the introducer needle was advanced approximately $1 \mathrm{~cm}$ into the disc. After confirming the introducer needle position using anteroposterior (AP) and lateral views, the stylet was removed, and the wand was inserted through the introducer needle. The wand had a navigable tip that could be curved to the desired angles by rotating a control wheel on the handle. We introduced the wand, and it passed the midline of the targeted disc. Using the control wheel, we placed the tip of the wand into the center of the posterolateral portion of the herniated disc. Before the radiofrequency current was applied, motor nerve stimulation was used to confirm that the tip of the wand was not close to the nerve root. Then, the radiofrequency current $\left(265 \mathrm{~V}_{\mathrm{rms}}\right)$ was applied to the herniated portion of the disc using an RF generator (L'DISQ $^{\circledR}, \mathrm{U}$

\& I Co., Uijeongbu, South Korea). During the decompression, the tip of the wand was continuously moved back and forth to increase the ablated disc volume. The 5-second ablation was performed 60 times. The procedural duration was 30 minutes. After the procedure, the patient reported no adverse effects. One week after the decompression procedure, the patient's pain score had reduced from 7 to 2 . At the 1-, 3-, and 6-month follow-ups, the patient's NRS pain score was 1. At his 2-year follow-up after the procedure, he reported that his radicular pain was sustained at an NRS score of 1 , and no oral medications or procedures were required.

Patient B was a 39-year-old man who visited the spine center at the University Hospital with pain in the right buttock, posterior thigh, and calf, which had lasted for 15 days. His NRS score was 8 out of 10 , and he could neither sit nor stand due to severe pain. The pain was piercing and tingling. Upon physical examination, he had a positive SLRT result at 30 degrees on the right side. His MRC score for the right extensor hallucis longus and plantar flexor was 4. Hyperalgesia and hyperesthesia 
were observed at the right S1 dermatome. On lumbar MRI, a large right central intervertebral disc extrusion was observed at the L5-S1 intervertebral disc (Figure 2B). Before his visit to our hospital, the patient had received 3 transforaminal and 2 interlaminar ESIs. However, no pain reduction was observed after each injection. We performed a percutaneous disc decompression under the guidance of fluoroscopy using the device and techniques that were used for patient A (Figure 2B). Post-procedural adverse effects were not observed. One week after the procedure, the patient's pain had reduced with a change in the NRS score from 8 to 1. At the 1-, 3-, and 6-month follow-ups, as well as the 2-year follow-up, the patient reported that his pain had completely resolved. Additionally, his motor deficits were nearly completely recovered.

\section{Discussion}

We reported two cases of successfully managed lumbosacral radicular pain that was due to large intervertebral disc herniations that were not responsive to repeated ESIs. Both patients showed excellent outcomes even after 2 years following the procedure.

Unlike with other wands used in disc compression, the wand in the navigable percutaneous disc decompression device can be curved as required by the interventionist. ${ }^{6}$ Therefore, its advantage is that the wand tip can get close to the herniated disc. Although a direct comparison between the outcomes of the navigable percutaneous disc decompression device and other disc compression devices has not been conducted, the navigable percutaneous disc decompression device may remove herniated portions of the disc more effectively.

In 2012, Hong et $\mathrm{al}^{11}$ investigated the safety of disc decompression using the navigable percutaneous disc decompression device. They found that the device increased the body's temperature by up to $13.25^{\circ} \mathrm{C}$ in discs tested in human cadavers. In the outer annulus, the increase in temperature was $<1^{\circ} \mathrm{C}$. Therefore, they reported that the structures outside the disc, such as the nerves and meninges, are not damaged by the use of L'DISQ $^{\circledR}$.

Three studies have reported the therapeutic outcomes of the navigable percutaneous disc decompression device on pain caused by lumbar spine disorders. ${ }^{6,10,12}$ In 2011, Lee et $\mathrm{al}^{6}$ performed percutaneous disc compression in 27 patients using the navigable percutaneous disc decompression device. They recruited patients with radicular pain whose nerve roots were compressed by a herniated disc. Their average visual analog scale (VAS) score had reduced from 7.08 at pretreatment to 1.84 at 24 weeks post-procedure. In 2015, Lee et $\mathrm{al}^{12}$ conducted the procedure with the navigable percutaneous disc decompression device in 20 patients with axial chronic discogenic back pain. At 48 weeks after the procedure, the average VAS score had changed from 7.55 at pretreatment to 3.60. In 2019, Ceylan et al $^{10}$ recruited 209 patients with HLD and a spinal canal with an axial diameter greater than $50 \%$. The average VAS score reduced from 7.28 at pre-treatment to 3.03 at the 1-year follow-up. The overall satisfaction rate was approximately $80 \%$. In these three previous studies, no major complications developed. However, disc decompression procedures are more invasive than ESIs. The navigable percutaneous disc decompression device can damage normal discs, not only herniated or pathological discs. Therefore, we recommend its use for patients with HLD whose radicular pain is refractory to repeated ESIs. However, in all the previous studies, the authors did not administer ESIs before the disc decompressions using L'DISQ.

In conclusion, our study is the first to show that percutaneous disc decompression with the navigable percutaneous disc decompression device may be useful for controlling lumbosacral radicular pain that is unresponsive to repeated ESIs. Our study is limited, as it involved only two cases. Further studies that involve more cases are required to elucidate the effects of the navigable percutaneous disc decompression device. In addition, studies for clarifying the indications of the navigable percutaneous disc decompression device should be conducted in the future.

\section{Funding}

The present study was supported by a National Research Foundation of Korea grant funded by the Korean government (Grant no. NRF-2019R1F1A1061348).

\section{Disclosure}

The authors have no conflicts of interest to report.

\section{References}

1. Jang SH, Chang MC. Follow-up of at least five years after lumbar transforaminal epidural steroid injection for radicular pain due to lumbar disc herniation. Ann Palliat Med. 2020;9(1):116-118. doi:10.21037/apm.2020.01.05

2. Ruschel LG, Agnoletto GJ, Aragão A, Duarte JS, de Oliveira MF, Teles AR. Lumbar disc herniation with contralateral radiculopathy: a systematic review on pathophysiology and surgical strategies [published online ahead of print, 2020 Apr 13]. Neurosurg Rev. 2020. doi:10.1007/s10143-020-01294-3 
3. Takahashi N, Yabuki S, Aoki Y, Kikuchi S. Pathomechanisms of nerve root injury caused by disc herniation: an experimental study of mechanical compression and chemical irritation. Spine (Phila Pa 1976). 2003;28(5):435-441. doi:10.1097/01.BRS.0000048645.33118.02

4. Kim SJ, Park SM, Cho YW, et al. Changes in expression of mRNA for interleukin- 8 and effects of interleukin- 8 receptor inhibitor in the spinal dorsal horn in a rat model of lumbar disc herniation. Spine (Phila Pa 1976). 2011;36(25):2139-2146. doi:10.1097/BRS.0b013e $31821945 \mathrm{a} 3$

5. Tak HJ, Jones R, Cho YW, Kim EH, Ahn SH. Clinical evaluation of transforaminal epidural steroid injection in patients with gadolinium enhancing spinal nerves associated with disc herniation. Pain Physician. 2015;18(2):E177-E185.

6. Lee SH, Derby R, Sul D, et al. Efficacy of a new navigable percutaneous disc decompression device (L'DISQ) in patients with herniated nucleus pulposus related to radicular pain. Pain Med. 2011;12 (3):370-376. doi:10.1111/j.1526-4637.2011.01064.x

7. Faciszewski T, Winter RB, Lonstein JE, Denis F, Johnson L. The surgical and medical perioperative complications of anterior spinal fusion surgery in the thoracic and lumbar spine in adults. A review of 1223 procedures. Spine. 1995;20:1592-1599. doi:10.1097/00007632199507150-00007
8. Andersson GB, Brown MD, Dvorak J, et al. Consensus summary of the diagnosis and treatment of lumbar disc herniation. Spine. 1996;21:75S-8S. doi:10.1097/00007632-199612151-00009

9. Rahimzadeh P, Imani F, Ghahremani M, et al. Comparison of percutaneous intradiscal ozone injection with laser disc decompression in discogenic low back pain. J Pain Res. 2018;11:1405-1410. doi:10.2147/JPR.S164335

10. Ceylan A, Aşık İ. Percutaneous navigable intradiscal decompression in treatment of lumbar disc herniation: a single-center experience. Turk J Med Sci. 2019;49(2):519-524. doi:10.3906/sag-1805-187

11. Hong YK, Derby R, Wolfer LR, et al. An assessment of a new navigatable percutaneous disc decompression device (l'DISQ) through histologic evaluation and thermo-mapping in human cadaveric discs. Pain Med. 2012;13(8):1000-1003. doi:10.1111/j.15264637.2012.01447.x

12. Lee SH, Derby R, Sul D, et al. Effectiveness of a new navigable percutaneous disc decompression device (L'DISQ) in patients with lumbar discogenic pain. Pain Med. 2015;16(2):266-273. doi:10.1111/ pme. 12603

\section{Publish your work in this journal}

The Journal of Pain Research is an international, peer reviewed, open access, online journal that welcomes laboratory and clinical findings in the fields of pain research and the prevention and management of pain. Original research, reviews, symposium reports, hypothesis formation and commentaries are all considered for publication. The manuscript management system is completely online and includes a very quick and fair peer-review system, which is all easy to use. Visit http:// www.dovepress.com/testimonials.php to read real quotes from published authors. 